\title{
Das EU-Umweltzeichen zwischen Mauerblümchen und Blumenwiese
}

\author{
Die Aktivitäten zum Umweltmanagement \\ (EMAS) und zur Umweltkennzeichnung (EU- \\ Umweltzeichen) sind der Europäischen Kom- \\ mission zufolge wichtige Maßnahmen auf \\ dem Weg zu einer nachhaltigen Entwicklung. \\ Welche Rolle wird in Zukunft das EU-Umwelt- \\ zeichen spielen? \\ Von Frieder Rubik und Dirk Scheer
}

$D^{i}$ ie Weiterentwicklung von EMAS und EU-Umweltzeichen steht auf der Agenda der Europäischen Kommission. Beide Verordnungen verpflichten die Kommission vor der Revision, eine Evaluation durchzuführen. Hierzu wurde ein europäisches Forschungskonsortium unter Beteiligung des IÖW beauftragt, diese beiden freiwilligen umweltpolitischen Instrumente einer Evaluation zu unterziehen und Vorschläge zur Weiterentwicklung vorzulegen.

\section{Das EU-Umweltzeichen als Baustein der nachhaltigen Entwicklung?}

Inwieweit gelingt es dem EU-Umweltzeichen (UZ), Produktions- und Konsummuster tatsächlich zu verändern und wie kann man die Umweltleistung dieses Systems verbessern? Welche fördernden und hemmenden Faktoren gibt es dabei? Welchen Beitrag leistet das EU-Umweltzeichen zu einer nachhaltigen Entwicklung und zur Erhöhung der Wettbewerbsfähigkeit von Unternehmen? Diesen Fragen ging das Projektteam nach. Zentrales Ziel des Vorhabens war die Beratung der Kommission bei der Revision der Europäischen Verordnung zum EUUmweltzeichen.

Hierzu wurde zunächst der Stand der fachwissenschaftlichen Debatte zu nationalen und internationalen Umweltzeichen nachgezeichnet. Darauf aufbauend wurden 81 Interviews mit Anwendern und Nichtanwendern des EU-Umweltzeichens, mit Fachbehörden und Nichtregierungsorganisationen in den 25 Mitgliedsstaaten durchgeführt. Die Schwerpunkte der Interviews lagen einerseits auf einer Einschätzung des Status Quo (unter anderem direkte und indirekte Effekte des EU-Umweltzeichens, Verhältnis zwischen nationalen und EU-Umweltzeichen, hemmende und fördernde Faktoren, Beitrag zur Wettbewerbsfähigkeit, Verhältnis zu Strategien einer nachhaltigen Entwicklung) sowie andererseits auf einer Benennung und Beurteilung von
Instrumenten und Optionen zur Fortentwicklung des Umweltzeichens. Ergänzend wurden einige Fallstudien durchgeführt. Zur Vertiefung der Erkenntnisse und Vorstellung erster Vorschläge wurde im September 2005 ein Workshop in Brüssel veranstaltet. Eine detaillierte Ergebnisdarstellung ist im Rahmen dieses Artikels nicht möglich (1). Im Folgenden werden daher ausgewählte und als interessant eingeschätzte Ergebnisse präsentiert.

\section{Umweltzeichen - ökologisch leistungsfähig, aber Marktkräfte unzureichend}

In Bezug auf direkte Effekte, das heißt ökologische Verbesserungen, die über die direkte Anwendung von Umweltzeichen initiiert werden, bestätigt sich die in der Literatur oftmals geäußerte These, dass die alleinigen Marktkräfte nicht ausreichen, mittels Umweltzeichen eine umfassende Markttransformation in Richtung der Herstellung von ökologischen Produkten und eines nachhaltigen Konsumverhaltens zu erreichen. Kurz: Der Nachfragesog gilt als unzureichend. Die Umfrage hat verifiziert, dass viele Unternehmen ihre Produkte nicht mit Umweltzeichen zertifizieren lassen, obwohl diese Produkte die Kriterien erfüllen würden. Die Hälfte der befragten Unternehmen, die sich nicht am Umweltzeichen beteiligen, war über die Kriterienanforderungen und -inhalte gut informiert. Eine Mehrheit dieser Befragten wies darauf hin, dass etwa 80 bis 95 Prozent ihrer Produkte die Kriterien erfüllen würden. Dies ist ein deutlicher Hinweis darauf, dass die Marktkräfte zu schwach sind, Unternehmen zur Zertifizierung ihrer Produkte mit Umweltzeichen zu bewegen.

Wie sieht es nun mit der eigentlichen Zielsetzung von Umweltzeichen, nämlich der Verbesserung der ökologischen Performance, aus? Zunächst bleibt festzuhalten, dass generell ein erheblicher Mangel an empirischen Daten zur Bewertung der durch Umweltzeichen initiierten ökologischen Verbesserung existiert. Auch die Befragung konnte keine absoluten quantitativen Daten zur Umweltverbesserung generieren. Dies liegt darin begründet, dass Unternehmen diesbezüglich kaum systematische Daten erfassen und erheben. Dennoch konnten qualitative Aussagen gewonnen werden. Befragt danach, wie wichtig die Verbesserung der ökologischen Leistungsfähigkeit bei der Entscheidung zur Einführung des europäischen Umweltzeichens war, äußerten über zwei Drittel, dass dies sehr oder ziemlich wichtig gewesen sei. Darüber hinaus wies ungefähr jeder zweite Befragte der EU-Blume-Zeichennehmer darauf hin, dass das europäische Umweltzeichen einen deutlich positiven Ein- $\Rightarrow$ 
fluss in den Bereichen Luft- und Wasseremissionen sowie Abfall und Recycling gehabt habe. Ökologische Verbesserungen im Bereich Lärm- und Geruchsbelästigung wurden hingegen von nur rund einem Viertel der Befragten konstatiert.

Vertieft wurde der Themenbereich ökologische Performance durch Fragen bezüglich der Aufstellung von ökologischen Zielsetzungen im Unternehmen. Ungefähr die Hälfte aller Unternehmensbefragten erklärte, dass ihr Unternehmen in allen beziehungsweise in den meisten Bereichen quantitative Umweltziele für die ökologische Verbesserung der Produkte formuliert. 41 Prozent der UZ-Teilnehmer und 55 Prozent der UZ-Nichtteilnehmer erklärten, dass ihr Unternehmen regelmäßig die ökologische Leistungsfähigkeit ihrer Produkte misst. Erstaunlich ist allerdings auch, dass eine systematische Erfassung der Leistungsfähigkeit von rund einem Drittel der Unternehmen mit einem Umweltzeichen nicht gemacht wird. Hier stellt sich die Frage, ob ein Umweltzeichen die Setzung von internen Umweltzielen ersetzt. Bezüglich des Einflusses der EU-Blume auf dieses interne Benchmarking zeigte sich aber, dass das europäische Umweltzeichen für 42 Prozent der teilnehmenden Firmen maßgeblich zur Setzung interner Umweltziele beigetragen hat.

Der Einfluss der EU-Blume geht aber auch über die Unternehmensgrenzen hinaus. So erklärten etwa drei Viertel der zertifizierten Unternehmen, dass das europäische Umweltzeichen einen starken Einfluss auf die firmenbezogenen Lieferbedingungen gehabt habe. Bei den nicht teilnehmenden Firmen waren dies immerhin noch 43 Prozent. Auch war für fast 74 Prozent der Befragten EU-Blume-Teilnehmer das EU-Umweltzeichen bereits Thema im Rahmen ihrer Kommunikation mit kommerziellen Kunden. Bei der Kommunikation mit privaten Kunden wurde die EU-Blume bei rund zwei Dritteln thematisiert. Die Befragung zeigte auch, dass Umweltzeichenkriterien oftmals als informeller Standard beziehungsweise Benchmark innerhalb einer Branche genutzt werden. Rund die Hälfte der nicht teilnehmenden Befragten erklärte, dass sie sich in allen oder fast allen Bereichen an Umweltzeichenkriterien orientieren. Auch sind den Befragten Unternehmen und Stakeholdern die politikbezogenen indirekten Effekte von Umweltzeichen bekannt. Dies wird in der Regel von den wirtschaftlichen Akteuren anerkannt und geschätzt.

\section{EU-Blume und/oder nationale Umweltzeichen?}

Eine in Europa seit langem und teils kontrovers geführte Diskussion thematisiert das Verhältnis zwischen dem EU-Umweltzeichen und nationalen Labels. Auch die Befragung spiegelte dies wider. Es konnte keine eindeutige Präferenz zugunsten des europäischen oder nationaler Umweltzeichen festgestellt werden. Auch wurde nicht eindeutig die These bestätigt, dass die nationalen Zeichen und das europäische Umweltzeichen miteinander konkurrieren.

Wie sollte der zukünftige Weg aussehen? Hier sprachen sich die Befragten eindeutig zugunsten von Harmonisierung und Ko- operation aus. Bei der Frage nach der Ausgestaltung von Harmonisierung gab es deutlichen Zuspruch für identische Institutionen, die die Zeichen vergeben, sowie bezüglich der Harmonisierung der Zeichenanforderungen für identische Produktgruppen und gleiche Anwendungsverfahren. Davon versprechen sich insbesondere die derzeitigen Nicht-Zeichennehmer einen vereinfachten Zugang zu mehr als einem Umweltzeichen, verbunden mit weniger Büroarbeit und einer Erleichterung, die Zeichenanforderungen zu erfüllen. Interessanterweise wurde das Kostenargument, das heißt Kostenreduktion beim Betrieb von Umweltzeichen, von den wirtschaftlichen Akteuren als deutlich weniger wichtig eingeschätzt als von den Stakeholdern.

\section{Fördernde und hemmende Faktoren}

Die Befragung befasste sich ausführlich mit der Identifizierung von unterstützenden und hemmenden Faktoren zur Steigerung der Effektivität des EU-Umweltzeichen sowie dessen Beitrag zur Wettbewerbsfähigkeit von Unternehmen. Auffallend ist, dass als unterstützende Faktoren vor allem markt- und wettbewerbsbezogene Aspekte, als hemmende Faktoren insbesondere administrative Aspekte genannt wurden. In Bezug auf Anspruchsgruppen haben die Befragten geäußert, dass sie gute Erfahrungen mit der Nutzung des europäischen Umweltzeichens hinsichtlich der Befriedigung spezieller Kundenwünsche, der Steigerung des Kundeninteresses und der Anerkennung als Marktführer gemacht haben. Darüber hinaus wurde die Verbesserung der internationalen Wettbewerbsfähigkeit und ein verbesserter Zugang zur öffentlichen Beschaffung positiv bewertet. Marktposition und -nachfrage sind demzufolge strategische Schlüsselbereiche, an denen Entwicklung und Design begleitender und unterstützender Maßnahmen für das europäische Umweltzeichen ansetzen sollte.

Dagegen beziehen sich die von den Befragten ausgemachten Hindernisse auf administrative, prozedurale und ressourcenbezogene Aspekte im Verlauf der Zertifizierung. So gilt der interne Dokumentations- und Bürokratisierungsaufwand als Hürde. Insbesondere die Beschaffung der notwendigen Dokumentation von Zulieferern bereitet vielen Schwierigkeiten. Zudem werden die durch die Erfüllung der Anforderungen verursachten zusätzlichen Kosten als wenig förderlich eingeschätzt. Interessanterweise gibt es unterschiedliche Einschätzungen zwischen Nichtzeichennehmern und Stakeholdern bezüglich unternehmensinterner Aspekte. Generell schätzen letztere diese Faktoren deutlich problematischer ein als die Unternehmen selbst. Zeitmangel, Mangel an personellen Ressourcen und Kompetenz oder fehlende Unterstützung wird von den bislang noch nicht mit der EU-Blume zertifizierten Firmen als geringes Problem eingeschätzt. Dies gilt gleichermaßen für Schwierigkeiten, die Kriterienanforderungen umzusetzen oder notwendige Unterlagen von den Zulieferern zu bekommen. Dass letzteres de facto dann doch ein Hindernis sein kann, zeigen allerdings die von den zertifizierten Betrieben gemachten Erfahrungen. 


\section{Optionen zur Weiterentwicklung}

Die Recherchen, Interviews und eigenen Überlegungen zur Fortentwicklung des EU-Umweltzeichens erbrachten eine Vielzahl operativer und strategischer Vorschläge, die im anstehenden Revisionsprozess ihren Niederschlag finden könnten. Die Nulloption, also das Einstellen des EU-Umweltzeichens, wurde mit Blick auf den zunehmenden europäischen und weltwirtschaftlichen Integrationsgrad und mit Blick auf den Trend hin zu umweltpolitischen Instrumenten mit größeren Freiheitsgraden als eine wenig sinnvolle Option angesehen, sowie als ein Rückfall in nationale Egoismen betrachtet. Verschiedene Optionen zur Fortschreibung des Europäischen Umweltzeichens konnten benannt werden, wobei jede Option mehrere Vorschläge umfasst.

Die Option einer institutionellen Reform zielt auf den gegenwärtigen institutionellen Rahmen des EU-Umweltzeichen und schlägt eine Neuordnung von Rechten, Pflichten und Entscheidungsbefugnissen vor. Dabei gilt die Maßgabe, dass die gesellschaftlichen Anspruchsgruppen in Analogie zum Blauen Engel quasi Eigner des EU-Umweltzeichens werden und die bisher bestehende letztendliche Entscheidungshoheit der Kommission entfällt. Die Vertreter der Mitgliedsstaaten sollten nur noch zwei Stimmen in dem Entscheidungsprozeß besitzen, die restlichen Stimmen sollten den Anspruchsgruppen zufallen. Ein Outsourcing und eine Privatisierung des Systems wurden ebenfalls untersucht, aber als kontraproduktiv angesehen.

Durch eine Veränderung der Rahmenbedingungen sollte in Zukunft eine noch stärkere Verknüpfung zwischen dem öffentlichen Beschaffungswesen und dem Umweltzeichen erfolgen. Einerseits sollte neben Fortbildungs- und Trainingsmaßnahmen auch die Möglichkeit eingeräumt werden, auf direktem Wege das Tragen eines Umweltzeichens in Ausschreibungsunterlagen zu verlangen. Andererseits könnte auch das Tragen eines Umweltzeichens zusätzliche Wertungspunkte in der Beurteilung eingereichter Angebote bei öffentlichen Ausschreibungen erbringen. Zudem sollte globalen Ereignissen, die wie die Fußballweltmeisterschaft direkt mit dem Beschaffungswesen verbunden sind, und den zugehörigen Symbolgütern mit Blick auf Umweltzeichen eine besondere Aufmerksamkeit geschenkt werden. So könnten für diese Symbolgüter Vergabegrundlagen erarbeitet und diese dann auch entsprechend beschafft werden werden.

Daneben wurden auch fiskalische Maßnahmen zur Veränderung der relativen Preise angeregt. Zum einen könnte das Mehrwertsteuersystem geändert und Produkte, die ein Umweltzeichen tragen, einem günstigeren Satz zugeordnet werden. Zum anderen könnte auch eine Verbindung zwischen Körperschaftssteuer und Umweltzeichen geschaffen werden, wie dies etwa in der Toskana der Fall ist: Unternehmen mit Produkten, die das EU-Umweltzeichen tragen, können dort Ermäßigungen bei der Körperschaftssteuer erhalten.

Eine Ausweitung des Umweltzeichens könnte durch die Erarbeitung von zusätzlichen Vergabegrundlagen für weitere Pro-

\section{„Vergabebedingungen, Validierungs- prozess, Rahmenbedingungen sowie das Marketing von EMAS und Umwelt- kennzeichnung sollten aufeinander abgestimmt werden."}

duktgruppen wie Symbolgüter oder strategisch bedeutsame Produktgruppen erreicht werden. Auch durch eine Verringerung sowie Fokussierung der Vergabekriterien, die bisher teilweise sehr verästelt und umfangreich sind, könnte das Umweltzeichen eine Ausweitung erfahren.

Einer Erhöhung der Bekanntheit unter Konsumenten, professionellen Einkäufern, Händlern, potenziellen Zeichennehmern und Stakeholdern kommt eine sehr große Bedeutung zu. Hierzu sollten einerseits direkte Aktivitäten wie Informationskampagnen, Co-Marketing mit derzeitigen Zeichennehmern und NGOs sowie Dialogforen angewandt werden. Andererseits wirken indirekte Maßnahmen wie Informationsmaterialien, Koordinierungseinrichtungen und Marktanalysen eher unterstützend. Unerlässlich ist auch ein Budgetposten im Haushalt der Kommission, aus dem diese Maßnahmen finanziert werden.

\section{Koexistenz verschiedener Zeichensysteme}

Angesichts der Globalisierung und des Binnenmarkts ist aus unserer Sicht die Koexistenz von nationalen und dem EU-Umweltzeichen fragwürdig. Grundsätzlich sollten die Mitgliedsstaaten veranlasst werden, entweder ihre Zeichensysteme zurückzuziehen oder 1:1 das EU-Umweltzeichen in ihren Vergabeanforderungen zu übernehmen. Politisch realistisch ist dies jedoch nicht. Deswegen sollten drei komplementäre Strategien verfolgt werden: Erstens - sofern nur ein Land Vergabebedingungen erarbeitet hat - Übernahme dieser durch das europäische Zeichen. Zweitens - wenn mindestens zwei Länder Vergabebedingungen erarbeitet haben - Festlegung europäischer Mindestbedingungen, die jedes Zeichensystem ergänzen darf, und dabei Möglichkeit der Verwendung beider Umweltzeichen. Drittens ein Verbot der Entwicklung nationaler Vergabekriterien in Bereichen, in denen bisher noch keine solchen erarbeitet wurden. Insgesamt würde das europäische System damit quasi in eine Art Dachmarke transformiert.

Für die Unterstützung von Bewerbern werden einerseits technische Maßnahmen zur Know-how-Bildung sowie anderseits finanzielle Anreize zur Verringerung der Kosten für die Zeichenbeantragung und -nutzung vorgeschlagen.

Eine direkte Ausweitung in Richtung eines Nachhaltigkeitszeichens wurde von uns als nicht sinnvoll betrachtet. Stattdessen sollten graduelle Modifizierungen durch die Einführung $\Rightarrow$ 
Abbildung 1: Abschließende Gewichtung und Beurteilung verschiedener Optionen

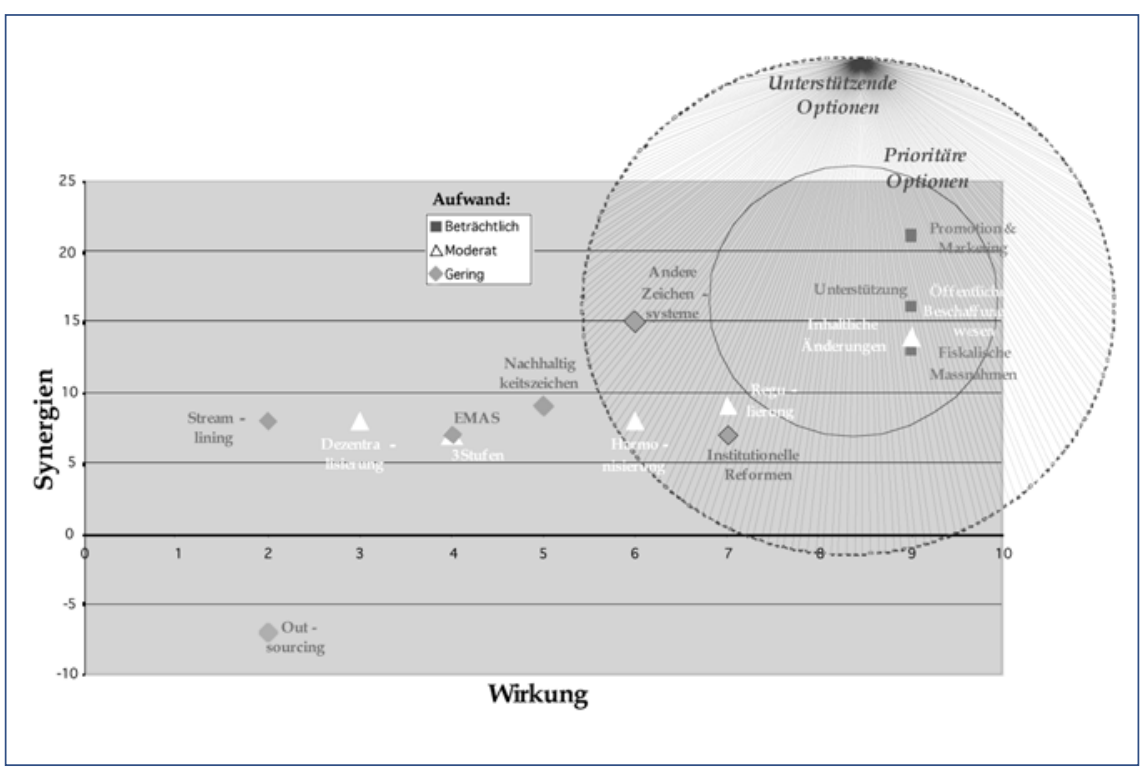

schätzung der Wirkungen, des Ressourcenaufwands sowie der Synergien erfolgte dabei innerhalb des Projektteams und konnte keiner externen Validierung unterzogen beziehungsweise quantifiziert werden. Verknüpft man nun diese Dimensionen in einer zweidimensionalen Matrix miteinander, so lassen sich prioritäre und unterstützende Optionen herausfiltern (Abbildung 1).

Dabei zeigt es sich sehr eindrücklich, dass Optionen, die beträchtliche Wirkungen haben können, Ressourcen benötigen. Gerade die prioritären Optionen Promotion/Marketing, Unterstützung der Antragsteller sowie fiskalische Maßnahmen werden beträchtliche Ressourcen benötigen. Einen etwas geringen Ressourcenverbrauch dürften die Mobilisierung des öffentlichen Beschaffungswesens sowie eine inhaltliche Änderung der Verga-

von Sicherheits- und Gesundheitsanforderungen eingeführt werden. Sie könnten langfristig die Weichen in Richtung eines Nachhaltigkeitszeichens stellen, indem die Aufmerksamkeit von Herstellern und Nachfragern auf einige in diesem Kontext wichtige Punkte gelenkt wird.

Sofern ein Unternehmen EMAS oder das Umweltzeichen nutzt, sollten die beiden Systeme sich gegenseitig unterstützen. Hierzu sollen Vergabebedingungen, Validierungsprozess, Rahmenbedingungen sowie Marketing aufeinander abgestimmt und koordiniert werden.

\section{Einführung eines „3-Stufen“-Systems}

Um eine intensivere und effektivere Integration zwischen EMAS, Umwelterklärungen (EPD) und Umweltzeichen zu erreichen, könnte eine noch weitergehende Verschmelzung verfolgt werden und so ein neues integratives System aufgebaut werden. Dabei wären drei Stufen zu unterscheiden: Die erste Stufe stellt das Umweltmanagementsystem EMAS dar, das auf Unternehmen und auf das Management der von dem Unternehmen ausgehenden Umweltbelastungen ausgerichtet ist. Die zweite Stufe zielt auf Möglichkeiten zur produktbezogenen Anerkennung von Umweltleistungen ab, indem Umwelterklärungen ermöglicht werden. Die dritte Stufe stellt die komparative Zertifizierung der Umwelteigenschaften eines Produkts im Vergleich zu denen der Wettbewerber dar. Dabei wird den ökologisch besten Produkten - auf Basis von Umwelterklärungen das Umweltzeichen verliehen werden.

Diese Optionen wurden jeweils qualitativ nach Maßgabe ihrer möglichen Wirkungen und der benötigten Ressourcen gewichtet, wobei ein dreistufiges ABC-Schema eingesetzt wurde. Daneben wurden die Synergien der einzelnen Optionen analysiert und ebenfalls qualitativ nach vier Stufen beurteilt. Die Ein- bekriterien und Produktgruppen nach sich ziehen. Umgekehrt gilt auch, dass die meisten „billigen“ Maßnahmen der Legitimation einer Reformbereitschaft dienen könnten, jedoch kaum mehr als Aktionismus darstellen werden. Ausnahmen sind jedoch institutionelle Reformen und eine Zusammenarbeit mit anderen Zeichensystemen, wie etwa dem Forest Stewardship Council (FSC), dem Marine Stewardship Council (MSC) oder Ökotex 100.

Man darf nun gespannt sein, ob und welche Anregungen die Europäische Kommission bei der Revision aufgreifen wird.

\section{Anmerkungen:}

(1) Der Bericht mit den gesamten Ergebnissen auch zur Evaluation von EMAS - steht unter http://ec.europa.eu/environment/ecolabel/documents/studies_en.htm zum Download bereit.

\section{AUTOREN + KONTAKT}

Dr. Frieder Rubik ist Leiter und Dirk Scheer ist wissenschaftlicher Mitarbeiter des Forschungsfelds „Ökologische Produktpolitik“ des IÖW im Büro Heidelberg.

IÖW Regional Büro Heidelberg, Bergstraße 7, D-69120 Heidelberg. Tel.: 06221/649160, E-Mail: frieder.rubik@ioew.de, dirk.scheer@ioew.de

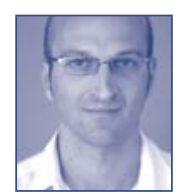


(c) 20I0 Authors; licensee IÖW and oekom verlag. This is an article distributed under the terms of the Creative Commons Attribution Non-Commercial No Derivates License (http://creativecommons.org/licenses/by-nc-nd/3.o/), which permits unrestricted use, distribution, and reproduction in any medium, provided the original work is properly cited. 